\title{
Adjuvant chemotherapy for breast cancer in patients with schizophrenia
}

\author{
MARK HWANG $^{1}$, MISHEL FARASATPOUR ${ }^{1}$, CAMPBELL D. WILLIAMS $^{1}$, JULIE A. MARGENTHALER $^{2}$, \\ KATHERINE S. VIRGO ${ }^{4}$ and FRANK E. JOHNSON ${ }^{1,3}$ \\ ${ }^{1}$ Department of Surgery, Saint Louis University Hospital; ${ }^{2}$ Department of Surgery, \\ Washington University Medical Center, St. Louis, MO 63110; ${ }^{3}$ St. Louis Veterans Affairs Medical Center, \\ St. Louis, MO 63106; ${ }^{4}$ Health Services Research, American Cancer Society, Atlanta, GA 30303-1002, USA
}

Received June 21, 2011; Accepted November 10, 2011

DOI: $10.3892 / \mathrm{ol} .2012 .560$

\begin{abstract}
The outcomes of treatment of physical illnesses are strongly affected by the presence of schizophrenia. We aimed to quantify the clinical course of schizophrenic breast cancer patients who were eligible for adjuvant chemotherapy to determine whether patients with this mental illness receive appropriate treatment for this physical illness. We searched the national Department of Veterans Affairs (DVA) computer database using computer codes for schizophrenia to identify patients who later developed breast cancer and were treated in DVA medical centers. Computer-based data were supplemented with chart-based clinical indicators. There were 55 subjects who appeared to be appropriate candidates for adjuvant systemic therapy. A number of these candidates were not offered postoperative endocrine or cytotoxic chemotherapy, while others refused treatment or were non-compliant. Behaviors typical of schizophrenic subjects, including hostility to caregivers, often disrupt their care. Schizophrenic patients often have advanced-stage cancer at diagnosis, often delay diagnosis and are frequently hostile towards healthcare workers. Many of these patients refuse therapy and/or are non-compliant.
\end{abstract}

\section{Introduction}

Breast carcinoma is the most common type of cancer (with the exception of non-melanoma skin cancer) and is the second leading cause of cancer-related mortality in American women. The incidence is approximately 123 per 100,000 women in the United States. The age-adjusted mortality rate in the US is approximately 24 per 100,000 women per year. The associ-

Correspondence to: Dr Frank E. Johnson, Department of Surgery, Saint Louis University Hospital, 3635 Vista Ave. at Grand Blvd, St. Louis, MO 63110, USA

E-mail: frank.johnson1@va.gov

Key words: adjuvant chemotherapy, breast cancer diagnosis, breast cancer treatment, schizophrenia ated lifetime risk for women is over $12 \%$, and over 2.5 million women currently living have a history of breast cancer in the US (1). With advancements in treatment, the mortality rate has declined over the last few decades.

There are various effective therapies available for breast cancer, and breast-conserving treatment is common (2). The use of adjuvant systemic chemotherapy for early-stage breast cancer produces a $23 \%$ or greater increase in disease-free survival duration and a $15 \%$ or greater increase in the overall lifetime survival rate (3). This has led to an improved quality of life with generally tolerable toxicities. Accumulating evidence suggests that breast cancer is a heterogeneous disease (4).

The early diagnosis and optimal treatment of breast cancer are essential (5). Delayed detection leads to poor outcomes and may be due to patient factors (including late presentation or lack of appreciation of the significance of a breast mass), physician factors (including misdiagnosis or delay in consultation requests) and/or societal factors (including inadequate access to care or insurance-related delays). All of these affect patients with schizophrenia. Recent investigations have focused on the physical health status of individuals with schizophrenia. Breast cancer is probably more common in schizophrenic patients than in the population as a whole. In their study, Bushe et al recently reviewed this topic (6). However, screening for common physical illnesses, such as breast cancer, is substantially less among such individuals than in the population as a whole.

Schizophrenia is a serious mental illness characterized by psychotic symptoms. It is typically chronic and causes characteristic abnormalities in perception, speech, behavior and thought. The diagnosis is based solely on clinical factors. Criteria for the diagnosis have been codified and published by the American Psychiatric Association (7) (Table I). Schizophrenia is believed to be the most disabling of mental disorders and places a large financial burden on patients, families and society. The incidence is low (15.2 per 100,000 individuals per year), but lifetime prevalence is relatively high $(7.2$ per 1,000$)(8,9)$. Approximately $1 \%$ of all populations worldwide studied thus far are afflicted. Since there are over 194,000 new patients diagnosed with breast carcinoma in the US each year and because the prevalence of schizophrenia in the US is more than $1 \%$ of the population, we estimate that 
Table I. Diagnostic criteria for schizophrenia.

Categories

Details/Explanation

Characteristic symptoms

Social/occupational dysfunction

Duration

Organic etiology exclusion

Schizoaffective and mood disorder exclusion

Pervasive developmental disorder relationship
Two or more symptoms during a one-month period: delusion, hallucinations, disorganized speech, disorganized/catatonic behavior and negative symptoms Marked decrease since onset of disturbance in work, relationships and self-care Continuous signs for at least six months

Includes: substance abuse or general medical conditions (including brain lesions, seizures and endocrinopathies)

Episodes of major depression, manic, or mixed episodes

Autism or other pervasive developmental disorders with characteristic symptoms of schizophrenia for $>1$ month is sufficient for the diagnosis of schizophrenia

All criteria are taken from the Diagnostic and Statistical Manual of Mental Disorders (7).

1,000-2,000 people with schizophrenia are diagnosed with breast cancer each year.

Providing medical care for unrelated physical disorders in schizophrenic patients presents unique challenges. Outcomes such as interactions between the mental illnesses and physical illnesses are of interest (10), but there are no studies pertaining to outcomes in populations of individuals with concurrent breast cancer and schizophrenia. Previously, we reported our analysis of the experience of the Department of Veterans Affairs (DVA) with the treatment of appendicitis in patients with schizophrenia (11). In this study, we aimed to describe the outcomes of management in a large population-based sample of schizophrenic patients with breast cancer in the DVA who were offered adjuvant chemotherapy. Surgeons are usually dominant figures in breast cancer care. We therefore suggest management strategies for such patients based on this analysis.

\section{Materials and methods}

Materials. Approval for this study was obtained from the institutional review board. The Patient Treatment File (PTF), the national archiving system for inpatient data in the DVA medical system, was accessed to retrieve data of interest.

The PTF was searched to identify patients with the International Classification of Diseases, Ninth Revision, Clinical Modifications (ICD-9-CM) diagnostic codes for schizophrenia or schizoaffective disorder (codes 295.00-295.85) who were also diagnosed with breast cancer (codes 174-174.9, 198.2, 198.81, 217, 233.0 and 611.72) during the fiscal years 1999-2005. The PTF identified appropriate patients based on these computer codes and yielded data concerning demographics, comorbid diseases and procedures.

To supplement the PTF data, we requested chart-based information from the DVA medical centers where patients had received their initial breast cancer treatment. These data included discharge summaries, progress notes, operative reports, medical oncology consultation summaries, psychiatry consultation notes and pathology reports. To obtain further information regarding the psychiatric history of each patient, we requested medical records predating admission for breast cancer; charts from the postoperative period were also obtained. Patients were classified as having received some type of mastectomy, lumpectomy or local excision as part of a curative-intent strategy if the records revealed breast carcinoma (codes 174-174.9, 198.2, 198.81, 217, 233.0 and 611.72). From this pool of patients we selected patients who appeared to be suitable candidates for adjuvant chemotherapy, according to the current National Comprehensive Cancer Network (12) and St. Gallen consensus conference standards (13).

In total, 27 (49\%) Caucasian, 19 (35\%) African-American, $3(5 \%)$ Hispanic and $6(11 \%)$ patients of other ethnicities were included in this study. There were 18 males $(33 \%)$ and 37 females $(67 \%)$. The mean age at the time of surgery was 53 years (standard deviation, 11 years). Of the 44 patients for whom living conditions were documented, 24 (55\%) were no longer living independently (i.e., they were living in the homes of family members, assisted living facilities, group homes, mental institutions or nursing homes), 17 (39\%) were independent and $3(7 \%)$ were homeless. Additionally, 10 of the $17(59 \%)$ schizophrenic patients living independently at the time of breast cancer diagnosis for whom data were available had been institutionalized within two years prior to their breast cancer treatment.

The majority of our patients (29/55 patients, 53\%) were diagnosed with paranoid schizophrenia (7) (Table I). Other subtypes of schizophrenia included undifferentiated $(9 / 55$ patients, $16 \%)$, residual $(2 / 55$ patients, $4 \%)$ and catatonic schizophrenia $(1 / 55$ patients, $2 \%)$. Patients with schizoaffective disorder $(15 / 155,27 \%)$ were also included. Of the 48 patients for whom the antipsychotic drug regimen was specified, 13 were taking a typical antipsychotic agent (such as thioridazine or haloperidol), 35 were taking an atypical antipsychotic agent (such as risperidone or clozapine), 5 were taking both types of drugs and 3 were on no antipsychotic drugs. The two most common outpatient medications administered were olanzipine (an atypical agent used by $17 / 48$ patients, $35 \%$ ) and risperidone (an atypical agent used by $12 / 48$ patients, 
Table II. Patient behaviors characteristic of schizophrenia observed in our subjects $(n=55)$.

\begin{tabular}{|c|c|c|}
\hline & $\mathrm{n}$ & $\%$ \\
\hline Unwarranted disruptive behavior & 7 & 13 \\
\hline $\begin{array}{l}\text { Unwarranted removal of intravenous } \\
\text { catheter by patient }\end{array}$ & 1 & 2 \\
\hline Failure to follow activity restrictions & 2 & 4 \\
\hline Requirement for restraints & 1 & 2 \\
\hline Self-mutilation & 1 & 2 \\
\hline Unwarranted refusal to eat & 2 & 4 \\
\hline Other adverse behaviors ${ }^{\mathrm{a}}$ & 6 & 11 \\
\hline
\end{tabular}

Mention of the behavior in the medical record was accepted as evidence that the behavior occurred and was significant enough in the opinion of a caregiver to warrant mention. Lack of mention of the behavior was taken as evidence that the behavior did not occur.

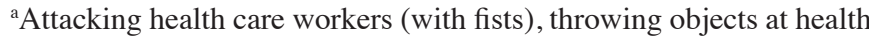
care workers, confusion about instructions from physicians leading to inability to appear for scheduled appointments.

$25 \%$ ). Of the 38 patients for whom documentation of ability to provide an accurate history was available, 15 patients $(39 \%)$ were described by a physician in the record as having a poor or questionable ability to provide a reliable history.

Methods and statistical analysis. We used simple descriptive statistics to portray the demographic data, clinical course and patient outcomes. Subjects were excluded from further review according to prespecified criteria. Mention of an abnormal thought or behavior in the medical record was accepted as evidence that it was observed by a caregiver. Lack of mention of an abnormal thought or behavior was accepted as evidence that it was not observed. Bivariate analyses were performed to determine the differences between schizophrenic patients with breast cancer and the general population using T-tests for continuous variables and Chi-square tests for categorical variables.

\section{Results}

Patient criteria. During the time period studied, 126 patients from 41 different VA facilities were identified by ICD-9-CM codes as having been diagnosed with schizophrenia and subsequently with breast cancer. We received data on 122 patients (97\%); 67 patients were excluded following the chart review as they did not have schizophrenia, did not have breast cancer, had a history of breast cancer predating the diagnosis of schizophrenia, did not have initial breast cancer treatment during the fiscal years 1999-2005, or had been deemed unsuitable for adjuvant chemotherapy due to comorbid conditions other than schizophrenia. The 55 remaining patients all had schizophrenia, later developed breast cancer that appeared to be curable, and were offered curative-intent treatment. All 55 patients appeared to be candidates for adjuvant chemotherapy. They were deemed evaluable and form the basis for this report.
Table III. Evidence of disordered thought observed in our subjects $(\mathrm{n}=55)$.

\begin{tabular}{|c|c|c|}
\hline Symptoms & $\mathrm{n}$ & $\%$ \\
\hline Confusion & 13 & 23 \\
\hline Lack of insight & 17 & 31 \\
\hline Auditory hallucinations & 27 & 49 \\
\hline Visual hallucinations & 17 & 31 \\
\hline Non-auditory/non-visual hallucinations & 4 & 7 \\
\hline Delusions & 26 & 47 \\
\hline Paranoia & 36 & 65 \\
\hline Abnormal affect & 25 & 45 \\
\hline Suspiciousness & 20 & 36 \\
\hline Ideas of reference & 14 & 25 \\
\hline Looseness of associations & 9 & 16 \\
\hline Thought blocking ${ }^{\mathrm{a}}$ & 7 & 13 \\
\hline
\end{tabular}

${ }^{a}$ Thought blocking (self-interruption of speech before completion) reflects disordered thought. Mention of the disordered thought in the medical record was accepted as evidence that it had been observed and was significant enough in the opinion of the caregiver to warrant mention. Lack of mention of the abnormal thought was taken as evidence that it was not observed.

Patient characteristics and behaviors. Our patient population comprised various ethnicities. Additionally, of the 44 patients whose living conditions were recorded, over half were not independent. Patients included were diagnosed with either paranoid or other subtypes of schizophrenia.

Many patients exhibited behaviors characteristic of schizophrenia during chemotherapy sessions and surgery (Table II). Classic evidence of disordered thought was often noted (Table III). Details concerning hallucinations and delusions were not recorded consistently; certain hospitals had recorded them in detail and others appeared to have recorded only the most notable incidents. For example, 5 of the 55 (9\%) had homicidal ideation documented in the medical record; $17 / 55(31 \%)$ had documented suicidal ideation; and 12/55 patients (22\%) had previously attempted suicide. Ten of the 55 patients $(18 \%)$ had verbally abused $\geq 1$ healthcare worker during $\geq 1$ hospital admission and 7 (13\%) patients had physically assaulted $\geq 1$ caregiver during $\geq 1$ hospital admission.

Fourteen of the $35(40 \%)$ patients for whom staging data were available had TNM stages III-IV; at least 5 of these 14 (38\%) patients had ignored obvious signs of breast cancer (mass and/or ulceration noted by patient) for six months to nine years; while 4 (29\%) other patients with biopsy-proven cancer had delayed treatment for $>3$ months. In the 25 patients for whom the tumor size was recorded, $17(68 \%)$ were $>2 \mathrm{~cm}$ (mean $5.8 \mathrm{~cm}$ ).

Among the 55 patients who appeared to be candidates for adjuvant cytotoxic therapy, there were 46 for whom adequate data on this option were available; only 39/46 (85\%) were offered postoperative cytotoxic chemotherapy; 12 of these $39(31 \%)$ patients refused or were non-compliant. Among the 55 patients who appeared to be candidates for adjuvant 
endocrine therapy, there were 38 for whom adequate data on this option were available. Only 25/38 (66\%) were offered postoperative endocrine therapy and 8 of these 25 (32\%) patients refused or were non-compliant. Refusal to take antipsychotic medication was noted in the medical record of 25 of our 55 (45\%) patients, but no statistically significant correlation was observed between refusal to take antipsychotic medications and refusal to accept adjuvant cytotoxic or endocrine chemotherapy (Chi-square analysis). However, suicidal ideation, homicidal ideation and a history of assaulting health care workers were statistically significantly correlated with non-compliance with adjuvant chemotherapy $(\mathrm{p}<0.05)$. History of substance abuse was statistically significantly correlated with non-compliance with endocrine therapy $(\mathrm{p}<0.05)$.

\section{Discussion}

Schizophrenia is a chronic, debilitating illness of unclear etiology. The phenotype is diverse, complex and often frightening to the afflicted as well as their caregivers. Modern genetic studies indicate that mutations in genes controlling neurodevelopmental sequences are etiologic factors (14-18). Environmental factors are also likely to be involved $(16,17)$. The anatomical, physiological and biochemical mechanisms suggested by genetic data are penetrant in neural structures, conform to what is known about the pathophysiology of schizophrenia derived from non-genetic investigations, and are concordant with the effects of these candidate genes (15). Schizophrenia is estimated to account for $1.1 \%$ of the disability-adjusted life-years worldwide (8). The economic burden of schizophrenia is estimated to be $\$ 30-40$ billion in the United States annually (19). It imposes a large burden on the affected individual, family members and society. Individuals with schizophrenia suffer appalling abuse throughout the world when under care for a physical illness such as breast cancer (20). Recent reports have documented widespread 'neglect, abandonment, indignity, cruel and inhuman treatment, and punishment' of schizophrenic subjects in spite of relevant international law, recommendations from international organizations and statements from various commissions, professional groups and government agencies (21).

Schizophrenia is fundamentally a psychotic disorder that interferes with the understanding of disease processes and leads to non-compliance with treatments. The majority of patients have a paranoid component to their illness that further hinders compliance with care. Decreased perception of pain and/or an altered expression of the emotional response to pain are well documented (22). Even in wealthy countries, those with major mental illnesses have a mean life expectancy approximately 25 years shorter than the population as a whole. Suicide, homicide and similar causes account for approximately $40 \%$ of the increased mortality rate although medical illnesses, including myocardial infarction, cancer and infectious disease are also estimated to play a role in this increased rate. Currently, available antipsychotic drugs reduce the mortality rate; however, patients must ingest them to achieve this outcome (23). The mortality rate due to breast cancer in schizophrenic patients is significantly higher than that in the general population, as is true for most types of cancer (24). The dimensions of this effect in many common medical conditions have not been quantified.
Thus, there is limited awareness of strategies to provide good clinical care for common physical disorders in these patients.

In 2006, there were an estimated 39.5 million hospitalizations in the US, according to the Agency for Healthcare Research and Quality (25). Approximately 1.4 million of these hospitalizations were due to mental illness; 7.1 million involved individuals with a mental illness in addition to the physical problem which was the primary reason for hospitalization. Schizophrenia and other psychoses without physical illness accounted for 381,000 hospital admissions. The cost of treating mental illnesses in the US was estimated to be $\$ 58$ billion/year in 2006, making it one of the most costly conditions (26).

A recent review of schizophrenia with answers to common clinical questions has been published (9). Such patients often have advanced-stage cancer at diagnosis, delay diagnosis, refuse treatment, fail to comply with adjuvant chemotherapy regimens and/or exhibit hostility to healthcare workers. Patients with schizophrenia have quantifiable abnormalities in their perception of pain and report pain differently than patients without schizophrenia (22). Similar abnormalities in other perceived sensations (including taste and smell) are also known. The incorrect interpretation of other symptoms and signs (presence of a breast mass, for example) appears to lead to delays in seeking medical attention, delayed diagnosis and suboptimal treatment.

In our subjects, certain presenting features, such as an abnormal screening mammogram (present in $74 \%$ of our patients), are similar to those in the general population of breast cancer patients. However, our patients had high rates of palpable breast mass (present in $80 \%$ of our patients), nipple retraction (present in 30\%) and nipple discharge (present in $11 \%$ ) compared to non-schizophrenic subjects with breast cancer, providing further evidence that schizophrenic patients do not report symptoms as promptly as non-schizophrenic ones. Signs of advanced breast cancer at diagnosis may be related, in part, to the fact that many or most schizophrenic patients see primary care doctors much less frequently than they see psychiatrists, who focus on the control of the mental illness rather than the diagnosis of physical diseases such as cancer. The delay may be caused by patient factors (such as confused thoughts, illicit drug abuse, altered perception of the significance of evident breast lesions and abnormal perception of pain), physician factors (such as inattention to breast symptoms, misdiagnosis and delay in consultation requests) and/or societal factors (such as inadequate access to care or insurance-related delays in treatment). Although the inability to access healthcare is a significant reason for schizophrenic patients not receiving the necessary treatment, all of the patients included in this study were eligible for care in the DVA system.

The majority $(85 \%)$ of our patients received some type of mastectomy rather than lumpectomy (15\%). Mastectomy carries a higher risk of complications than lumpectomy plus an axillary procedure (27). Moreover, the procedure is contrary to the current trend towards breast-conservation management. Adverse events contributing to the markedly higher risk of premature mortality are known to occur during hospitalization. In a large population-based study, Daumit et al estimated that schizophrenic patients have more than a two-fold higher 
risk of several major complications during hospitalization compared to those without schizophrenia (28). Complications include nosocomial infections, decubitus ulcers, postoperative respiratory failure, deep venous thrombosis, sepsis and hemorrhage. Such complications were observed in our patients prior to adjuvant chemotherapy commencing and often delayed diagnosis and treatment. These adverse events and the difficulties in receiving adjuvant systemic therapy presumably explain the increased mortality rate observed in schizophrenic breast cancer patients.

Our study revealed both a low rate of recommendation of adjuvant chemotherapy by physicians and a low rate of compliance by schizophrenic patients. There are numerous mechanisms that lead schizophrenic breast cancer patients to delay their care. For example, patients may believe that their doctors intend to harm them and often do not trust the healthcare system. Some of the patients in our series believed that 'cleansing teas', meditation and other unorthodox therapies were more likely to cure them than conventional methods. Paranoia and delusions often led to violence directed towards healthcare workers, as was observed in 10/55 (18\%) of our patients. We did not find a suitable published control group for our study. In the general population, use of adjuvant endocrine therapy as part of a curative-intent treatment plan for low-income breast cancer patients with medical insurance is notably low. Only $64 \%$ of patients reported by Kimmick et al had their prescriptions filled and the rate of adherence to the adjuvant treatment plan was $60 \%$ (29). Women older than 65 with early-stage, high-risk breast cancer (not selected for the presence of schizophrenia but with medical insurance) also frequently do not receive adjuvant chemotherapy (30).

There are, however, methodological limitations to our study. Patients were identified by computer codes for diagnoses of schizophrenia without the means to check whether these patients fit the diagnostic criteria established by the American Psychiatric Association. Another confounding factor that may account for adverse outcomes of therapy for breast cancer is the use of antipsychotic drugs, which may cause major complications, including obesity, diabetes, myocarditis, sedation, agranulocytosis, anticholinergic effects, neuroleptic-induced movement disorders (including the life-threatening neuroleptic malignant syndrome) and death (31). Our study is a retrospective analysis of cases; thus, criteria for diagnoses and reporting patterns for clinical features and treatment of outcomes were presumably not recorded consistently among our patients. In this report, we accepted the mention of a symptom, sign or complication as evidence of its presence unless stated otherwise. Similarly, we accepted absence of the mention of a particular variable as evidence of its absence unless stated otherwise.

Our study also has several strengths. To the best of our knowledge, this is the largest series on this topic in the literature. The DVA dataset from which the cases were selected is large, with approximately 25 million registered veterans. Among these, approximately five million receive care in DVA medical centers each year. Most DVA subjects are male, unlike those in most other databases. A comparison of female and male breast cancer patients using the National Cancer Institute's Surveillance, Epidemiology and End Results (SEER) data has been published (32). Sharma et al recently reported the experi- ence of the Imperial College Healthcare NHS Trust, a large British system based in London (33). Their aims and methods differed somewhat from ours. These authors concluded that schizophrenia does not affect the treatment of women with breast cancer. They acknowledge that their conclusions are not concordant with those of earlier reports. Our conclusions also differ from those of Sharma et al. The reasons for this discrepancy are speculative. The DVA system is enriched in schizophrenic subjects since it is a safety net source of care for poor veterans who are often homeless and/or unemployed. In general, individuals with schizophrenia, as is the case with individuals with other major mental illnesses, experience difficulty receiving indicated medical care (34). The patients in our series had access to care in the DVA system, although they may have had difficulty navigating it. Due to this difficulty, our analysis is likely to have underestimated the effect of preexisting schizophrenia on the process of breast cancer treatment.

We strongly recommend that clinicians caring for schizophrenic patients with a significant intercurrent physical illness such as breast cancer and known schizophrenia obtain a formal psychiatric evaluation of the patient. There are several reasons for obtaining such an evaluation. Having an independent expert opinion from a psychiatrist is essential in determining whether a patient is competent to make decisions about his/her own medical care. The risk to caregivers and other patients managing a schizophrenic patient is a matter of practical concern that a psychiatrist is trained to assess. The available evidence on this topic has been summarized by Friedman (35). Violence in those with serious mental illness is a non-negligible hazard even if the illness appears to be well controlled. The hazard increases markedly when other predisposing factors (particularly drug abuse) are present (36). The psychiatrist also provides advice on the need for a minder to protect the patient from self-harm. Even a short lapse in taking antipsychotic medications markedly increases the risk of hospitalization in schizophrenics (37). This is due to the recurrence of symptoms of thought disorder. The authors believe that a psychiatrist should be an integral part of the care team in such patients.

Our data show that patients with breast cancer and preexisting schizophrenia often present with late-stage cancer and often do not understand their disease or the necessary treatment. These patients delay or refuse adjuvant chemotherapy far more often than the population as a whole. They frequently do not cooperate well with caregivers and may be better served by radical surgery rather than breast-conserving treatment as an initial therapy.

The effect of major mental illness on the diagnosis and treatment of unrelated physical disease, as well as its effect on the outcome of treatment, is grossly under-researched and underreported. Further studies utilizing other data sets may clarify the effects of major mental illness on the diagnosis, treatment and course of unrelated physical disorders and provide guidance to clinicians caring for such patients.

\section{References}

1. Altekruse SF, Kosary CL, Krapcho M, et al: SEER Cancer Statistics Review 1975-2007. National Cancer Institute, Bethesda, MD. http://seer.cancer.gov/csr1975_2007/.

2. Sainsbury JR, Anderson TJ and Morgan DA: Clinical review: $\mathrm{ABC}$ of breast diseases. BMJ 321: 745-750, 2000. 
3. Gonzalez-Angulo AM, Morales-Vasquez F and Hortobagyi GN: Overview of resistance to systemic therapy in patients with breast cancer. Adv Exp Med Biol 608: 1-22, 2007.

4. Cianfrocca $\mathrm{M}$ and Gradishar W: New molecular classification of breast cancer. CA Cancer J Clin 59: 303-313, 2009.

5. Olivotto IA, Lesperance ML, Truong PT, et al: Intervals longer than 20 weeks from breast-conserving surgery to radiation therapy are associated with inferior outcome for women with early-stage breast cancer who are not receiving chemotherapy. J Clin Oncol 27: 16-23, 2009.

6. Bushe CJ, Bradley AJ, Wildgust HJ and Hodgson RE: Schizophrenia and breast cancer incidence: A systematic review of clinical studies. Schizophr Res 114: 6-16, 2009.

7. Frances A, Pincus HA, First MB, et al (eds): Diagnostic and Statistical Manual of Mental Disorders. 4th edition. Text revision. American Psychiatric Association, Washington, DC, 2000.

8. Saha S, Chant D, Welham J, et al: A systemic review of the prevalence of schizophrenia. PloS Med 2: 413-433, 2005.

9. Picchioni MM and Murray RM: Schizophrenia. Br Med J 335 91-95, 2007.

10. Burns T: Evolution of outcome measures in schizophrenia. Br J Psychiatry 191 (Suppl 50): S1-S6, 2007.

11. Cooke BK, Magas LT, Virgo KS, et al: Appendectomy for appendicitis in patients with schizophrenia. Am J Surg 193: 41-48, 2007.

12. Carlson RW, Allred DC, Anderson BO, et al: Breast Cancer Clinical Practice Guidelines in Oncology. www.nccn.org. J Natl Compr Canc Netw 7: 122-192, 2009.

13. Harbeck N and Jakesz R: St. Gallen 2007: Breast cancer treatment consensus report. Breast Care 2: 130-134, 2007.

14. McClellan JM, Susser and King M: Schizophrenia: a common disease caused by multiple rare alleles. Br J Psychiatry 190: 194-199, 2007.

15. Esslinger C, Walter $\mathrm{H}$, Kirsch $\mathrm{P}$, et al: Neural mechanisms of a genome-wide supported psychosis variant. Science 324: 605 , 2009.

16. Walsh T, McClellan JM, McCarthy SE, et al: Rare structural variants disrupt multiple genes in neurodevelopmental pathways in schizophrenia. Science 320: 539-543, 2008.

17. Allen NC, Bagade S, McQueen MB, et al: Systematic meta-analyses and field synopsis of genetic association studies in schizophrenia: the SzGene database. Nat Gen 40: 827-934, 2008

18. Cook EH and Scherer SW: Copy-number variations associated with neuropsychiatric conditions. Nature 455: 919-923, 2008.

19. Dixon L, Lyles A, Smith C, et al: Use and costs of ambulatory care services among Medicaid enrollees with schizophrenia. Psychiatr Serv 52: 786-792, 2001.

20. Kleinman A: Global mental health: a failure of humanity. Lancet 374: 603-604, 2009.

21. Minas H: Mentally ill patients dying in social shelters in Indonesia. Lancet 374: 592-593, 2009.
22. Talbott JA and Linn L: Reactions of schizophrenics to life-threatening disease. Psychiatr Q 5: 218-227, 1978.

23. Tiihonen J, Lonnqvist J, Wahlbeck K, et al: Eleven-year follow-up of mortality in patients with schizophrenia: a population-based cohort study (FINII study). Lancet 374: 620-627, 2009.

24. Tran E, Rouillon F, Loze JY, et al: Cancer mortality in patients with schizophrenia. Cancer 115: 3555-3562, 2009.

25. Saba DK, Levit KR and Elixhauser A: Hospital stays related to mental health, 2006. Statistical Brief 62. Healthcare Cost and Utilization Project. October 2008. Agency for Healthcare Research and Quality. (www.hcup-US.ahrq.gov/reports/statbriefs/sb62.pdf)

26. Soni A: The five most costly conditions, 1996 and 2006: estimates for the US civilian noninstitutionalized population. Agency for Healthcare Research and Quality. Medical Expenditure Panel Survey. Statistical Brief 248, July 2009. (www.meps.ahrq.gov/ mepsweb/data_files/publications/st248/stat248.pdf).

27. El-Tamer MB, Ward BM, Schifftner T, et al: Morbidity and mortality following breast cancer surgery in women: national benchmarks for standards of care. Ann Surg 245: 665-673, 2007.

28. Daumit GL, Pronovost PJ, Anthony CB, et al: Adverse events during medical and surgical hospitalizations for persons with schizophrenia. Arch Gen Psychiatr 63: 267-272, 2006.

29. Kimmick G, Anderson R, Camacho F, et al: Adjuvant hormonal therapy use among insured, low-income women with breast cancer. J Clin Oncol 27: 3445-3451, 2009.

30. Buist DS, Chubak J, Prout M, et al: Referral, receipt, and completion of chemotherapy in patients with early-stage breast cancer older than 65 years and at high risk of breast cancer recurrence. J Clin Oncol 27: 4508-4514, 2009.

31. Ray WA, Chung CP, Murray KT, et al: Atypical antipsychotic drugs and the risk of sudden cardiac death. N Engl J Med 360: 225-235, 2009

32. Anderson WF, Jatoi I, Tse J and Rosenberg PS: Male breast cancer: a population-based comparison with female breast cancer. J Clin Oncol 28: 232-239, 2009.

33. Sharma A, Ngan S, Nandoskar A, et al: Schizophrenia does not adversely affect the treatment of women with breast cancer: a cohort study. Breast 19: 410-412, 2010.

34. Bradford DW, Kim MM, Braxton LE, et al: Access to medical care among persons with psychotic and major affective disorders. Psychiatr Serv 59: 847-852, 2008.

35. Friedman RA: Violence and mental illness - how strong is the link? N Engl J Med 355: 2064-2066, 2006.

36. Fazel S, Langstrom N, Hjern A, et al: Schizophrenia, substance abuse, and violent crime. JAMA 301: 2016-2023, 2009.

37. Law MR, Soumerai SB, Ross-Degnan D and Adams AS: A longitudinal study of medication nonadherence and hospitalization risk in schizophrenia. J Clin Psychiatry 69: 47-53, 2008. 